\title{
Menschen im sozialen Raum
}

\section{Das Person-In-Environment-System hat unausgeschöpftes Potenzial}

\section{PETER PANTUČEK}

Prof. Dr. Peter Pantuček ist Studiengangsleiter Soziale Arbeit an der Fachhochschule Pölten in Österreich.

E-Mail peter@pantucek.com

\author{
Mit dem Person-In-Environment-System soll \\ eine ganzheitliche Einschätzung der Situation \\ von Menschen in problematischen Lebenslagen \\ gewonnen werden. Dabei spielen individuelle und \\ strukturelle Problemlagen ebenso eine wichtige \\ Rolle wie auch individuelle Ressourcen und \\ Unterstützungssysteme im sozialen Umfeld.
}

Vor mehr als 15 Jahren stellten in den USA die beiden Sozialarbeitswissenschaftler James N. Karls und Karin E. Wandrei erstmals den Entwurf eines Klassifikationssystems für die Soziale Arbeit vor (Karls/Wandrei 1992 und 1994).

Im deutschen Sprachraum wurde es u. a. durch Adler $(1998,2003)$ und Löcherbach (2010) rezipiert und vorgestellt. Eine deutsche Übersetzung der Formulare und der ersten Version des Manuals findet sich im Internet (www. pantucek.com, Karls/Wandrei 2007). Im Jahre 2008 wurde eine erneuerte Version des Person-In-Environment Systems veröffentlicht (Karls/O'Keefe).

Weder in den USA noch im deutschen Sprachraum fand das System in größerem Umfang den Weg in die Praxis. Der Vorstoß von Karls und Wandrei war ehrenvoll und bei genauerer Betrachtung auch durchdacht. Einer Verbreitung stand aber entgegen, dass die Systematik etwas abseits der gebräuchlichen quasi-intuitiven Kategorisierungen liegt und auf den ersten Blick in der Fallbearbeitung keine Vorteile und Erkenntnisgewinne verspricht.

Mit Widerstand auf der Ebene des Frontline-Social Work konnte also gerechnet werden. Gleichzeitig waren auch die möglichen Vorzüge auf der Ebene aggregierter Daten wenig herausgearbeitet und expliziert, so dass es für die Träger Sozialer Arbeit kaum attraktiv schien, sich die Mühen der Implementierung anzutun.

\section{Der Zugang des Person-In- Environment Systems}

Vier Faktoren werden im Person-InEnvironment System abgebildet:

- Faktor 1: Probleme in Rollen

- Faktor 2: Umwelt

- Faktor 3: Gesundheit (gemäß der Internationale Klassifikation der Krankheiten ICD10)

- Faktor 4: psychische Gesundheit (gemäß Diagnostic and Statistical Manual of Mental Disorders DSM IV)

Die Faktoren 3 und 4 sind nicht Gegenstand sozialer Diagnostik, kennzeichnen aber relevante Rahmenbedingungen. Das Person-In-Environment Systems verweist bei ihnen auf gängige Klassifikationssysteme - das ICD10 bei der physischen Gesundheit, das DSM IV bei der psychischen Gesundheit.

Bei den Faktoren 1 und 2 geht das Person-In-Environment System vorerst nicht von einer vorausgesetzten Normvorstellung, sondern von »Problemen « aus. Was hier als »Problem" verstanden wird, macht ein Satz im Manual deutlich: »Eine erfolgreiche Bewältigung sozialer Funktionen beinhaltet die Fähigkeit der KlientInnen, die Anforderungen des täglichen Lebens zu 


\section{Was versteht man eigentlich unter dem Person-In-Environment (PIE)?}

Zu Beginn eines Hilfeprozesses stellt die Einschätzung der Situation, der Ressourcen und der Probleme des Klienten einen bedeutsamen Schritt beim Case Management dar. Sie wird als Assessment (Einschätzung) bezeichnet und bildet die Grundlage für die Planung der gesamten Hilfe. Allerdings existierte lange Zeit hierfür in der Sozialarbeit kein einheitliches Klassifikationssystem. Anlass für die Entwicklung des PIE war das Fehlen eines solchen Klassifikationssystems für die systematische Identifizierung und Codierung von psychosozialen Problemen.

Das PIE-System wurde in erster Linie für Praktiker entwickelt, um Probleme, mit denen die Klienten die Sozialarbeiter konfrontieren, besser einordnen zu können und im Rahmen des Case Management Interventionen für jedes identifizierte Problem zu entwickeln. Dabei wird im PIE das Konstrukt der sozialen Rolle zugrunde gelegt, um Probleme der sozialen Funktionen zu erfassen. Das PIE bietet also eine Basis, um eine Klassifikation sozialer Probleme sowie eine Systematik sozialarbeiterischer Interventionen vorzunehmen und weiter zu entwickeln. Ein weiteres Ziel ist, den Praktikern der Sozialen Arbeit eine gemeinsame Sprache zur Verfügung zu stellen.

Das Klassifikationssystem PIE (Person-in-Environment) erfasst die sozialen Funktionen beziehungsweise sozialen Probleme der Klienten. Es dient damit der Diagnostik und Klassifikation sozialer Funktionsstörungen. Außerdem werden auch psychische Erkrankungen und Verhaltensprobleme sowie körperliche Erkrankungen integriert.

Quelle: Helmut Adler, Eine gemeinsame Sprache finden. Klassifikation in der Sozialen Arbeit - Ein Versuch: das Person-InEnvironment System (PIE). In: Blätter der Wohlfahrtspflege $7+8 / 1998$. meistern (zum Beispiel, sich Nahrung, Unterkunft und Transportmöglichkeit zu verschaffen) und die grundlegenden sozialen Rollen, die von den KlientInnen in ihrem unmittelbaren Bezugsfeld erwartet werden, ausreichend zu erfüllen." (Karls/Wandrei 2011)

Die erforderliche Komplexitätsreduktion gelingt, indem ein Filter genutzt wird, der auch in der Sozialarbeitspraxis wirksam ist. Gegenstand der Betrachtung ist, was als Problem definiert wird. Wo keine Problemdefinition, da keine Klassifizierung. So erspart man sich die Durchforstung von allen Faktoren, die für "gelingendes Leben« relevant sein könnten.
(2002:64). Weiters bietet das Raster des Person-In-Environment Systems beim Faktor 1 keine geeignete Heuristik zur Auffindung möglicherweise noch nicht thematisierter, aber doch bedrohlicher »covert problems « - es sei denn, man versteht die umfangreiche Auflistung von Rollen als Frageraster.

Eine weitere Schwäche war in der ersten Version bei der Abbildung von Ressourcen erkennbar, die nur über den »Coping Index", der neben dem Intensitäts- und dem Dauer-Index einem Problem zugeordnet wird, möglich war. Damit wird die Fähigkeit der Klientinnen und Klienten angegeben, »Probleme zu lösen und unabhängig zu

\section{"Die sfallunspezifische Arbeit, kann von dem Konzept des Person-in-}

\section{Environment profitieren«}

Ganz ohne Bezug auf Normen kommt allerdings auch dieser Zugang nicht aus. Er referiert beim Faktor 1 aber auf die vorfindlichen Rollenerwartungen und liefert so einen allgemein formulierten relativ und situativ anzuwendenden Maßstab.

Allenfalls wäre kritisierbar, dass der Faktor »Probleme in Rollen« zwar die Abbildung von nahezu allen Problemen der Lebensführung zulässt, mit denen Sozialarbeit zu tun hat, für eine $\mathrm{Ab}$ grenzung beispielsweise gegenüber psychologischer Beratung oder Psychotherapie jedoch wenig geeignet scheint, worauf beispielsweise Ehlerding hinweist handeln ..., sowie das Ausmaß des Vorhandenseins von Ich-Stärke, Einsicht und intellektueller Leistungsfähigkeit « (Karls/Wandrei 2007). Äußere Ressourcen wurden ebenso wenig erfasst, wie unspezifische Ressourcen - also solche, die (noch) nicht für die Bewältigung des bezeichneten Problems genutzt werden.

Im Manual 2nd Version (2008) wurde dieses Manko teilweise behoben. Ressourcen können nun auch dort klassifiziert werden, wo sie nicht unmittelbar mit relevanten Problemen im $\mathrm{Zu}$ sammenhang stehen. Hier interessiert vor allem der Faktor 2 des Assessments im Person-In-Environment System, der
Faktor »Umwelt «. Es wird die Qualität des Environments, in dem Personen ihr Leben führen, in den Blick genommen.

Karls und Wandrei nehmen als Grundlage für den Faktor 2 den Strukturvorschlag von Roland Warren (1963). Die von ihm genannten fünf Gemeinwesen-Funktionen können nur eingeschränkt von einer Community selbst organisiert und garantiert werden. Sie ist darin von anderen Gemeinwesen bis hinauf zur Ebene des Staates abhängig. Die fünf Funktionen nach Warren (1963) sind:

- Production-distribution-consumption

- Socialization

- Social control

- Social participation

- Mutual support

"... the definition of the community in terms of the systems that perform the major social functions having locality relevance leads to an emphasis on community functions rather than on community institutions. A conventional way of describing the related community phenomena is to consider the various institutional areas of the community: its economic institutions, its government, its educational institutions, its religious institutions, and perhaps its health and welfare, recreational, communicational, or other institutions. As noted, however, these institutional areas correspond only very loosely to the major locality-relevant functions. As already indicated, most of these functions are performed by a great variety of institu- 
tional auspices.« (Warren 1963:8)

So wird die Funktion »Socialization " (Vermittlung von Wissen, sozialen Werten, Verhaltensmustern) institutionell von Kindergärten und Schulen wahrgenommen, jedoch spielen auch die $\mathrm{Fa}$ milien und andere Akteure eine aktive Rolle im Prozess. Ähnlich bei »Social Control «: »The police and the courts are especially relevant in the performance of the social-control function by local government, but, as we shall see, many other social units, including the family, the school, the church, and the social agency, also play a large part.« (Warren 1963:10).

Im Gegensatz zu Warren referieren Karls und Wandrei nicht auf Funktionen, sondern auf Systeme und Institutionen der gesellschaftlichen Organisation. Sie folgen Warrens Strukturierung nur ungefähr und verlieren durch den Wechsel von »Funktion « zu »System« (hier näher dem Verständnis von »Funktionssystem « etwa bei Luhmann) die verwandtschaftlichen und nachbarschaftlichen Akteure weitgehend aus dem Blick.

Nicht zuletzt deshalb mussten sie als sechstes System das »System affektiver Unterstützung « hinzufügen: So bezeichnen sie die persönlichen Beziehungen, das »System von Freundschaften und Bekanntschaften, das einer Person individuelle soziale Unterstützung bietet « (Karls/Wandrei 2011:27). Die Bezeichnung als System "affektiver « Unterstützung ist ein Hinweis auf die Tendenz des Person-In-Environment Systems zur Psychologisierung.

Die verwandtschaftlichen, nachbarschaftlichen, freundschaftlichen, kollegialen Beziehungsnetze von Personen - ihr soziales Kapital im Sinne Bourdieus - leisten mehr als nur affektive Unterstützung, sie umfassen beispielsweise auch materielle Unterstützung, Information, lebensweltliche Beratung und so weiter. Eine einigermaßen detaillierte Auflistung der unterstützenden Funktionen des persönlichen Netzwerks findet sich beispielsweise bei Pearson (1997).

Darüber hinaus hat dieses Netzwerk nicht nur unterstützende Funktionen, sondern auch begrenzende, orientierende, erzieherische (»socialization « bei Warren) und die Funktion der sozialen Kontrolle. Hier findet sich also ein logischer Schwachpunkt des Person-InEnvironment Systems.
Die Entscheidung von Karls und Wandrei, Warrens Modell nicht komplett zu übernehmen, sondern stärker auf Systeme statt Funktionen zu fokussieren und nicht den Gesamtstatus, sondern »Probleme « in Zusammenhang mit Faktor 1 (»Probleme in Rollen «) in den Blick zu nehmen, eröffnet aber auch interessante Perspektiven. Diese werden vor allem dann sichtbar, wenn man nicht den Einzelfall, betrachtet, sondern die für ein Klassifikationssystem charakteristische Möglichkeit, Einzelfalldaten zu aggregieren. In Kombination mit Personenstandsdaten könnte so aufgewiesen werden, inwiefern der Zustand der Gemeinwesen-Infrastruktur Bewohnerinnen und Bewohnern mit ausgewählten Merkmalen Probleme bei der Lebensführung bereitet. Voraussetzung dafür wäre eine einigermaßen durchgängige Nutzung des Klassifikationssystems durch Sozialarbeiterinnen und Sozialarbeiter sowie die Zusammenführung und statistische Auswertung der Daten.

\section{Faktor Umwelt im Detail}

In das Person-In-Environment System wurden folgende Faktoren und Systeme aufgenommen und detailliert:

1. ökonomisches System und System der Grundversorgung

1.1 Ernährung

1.1.1. Fehlen regelmäßiger Versorgung mit Lebensmitteln

1.1.2. inadäquate Versorgung

1.1.3. dokumentierte Fehlernährung

1.1.4. andere

1.2 Unterkunft

1.2.1. keine Unterkunft

1.2.2. Substandard oder inadäquate Unterkunft

1.2.3.andere

1.3. Arbeit

1.3.1. Arbeitslosigkeit

1.3.2. Unterbeschäftigung

1.3.3. unangemessene Arbeit

1.3.4. andere

1.4. ökonomische Ressourcen

1.5.Verkehr

2. Bildungssystem

2.1. System der Ausbildung

3. Rechtssystem

3.1. rechtsstaatliches System

4. Gesundheits-, Sicherheits- und Sozialsystem

4.1. Gesundheit, geistige Gesundheit

\subsection{Sicherheit}

4.3. Sozialdienste

5. Selbstorganisationssystem

5.1. religiöse Gruppen

5.2. andere Gruppen im Gemeinwesen 6. System emotionaler Unterstützung

6.1. emotionale Unterstützung

Bei jedem System wird noch nach Diskriminierung gefragt: Besteht eine Diskriminierung im Zugang wegen

- Alter

- Ethnizität, Hautfarbe, Sprache

- Religion

- Geschlecht

- sexueller Orientierung

- Lebensstil

- mangelnder Staatsbürgerschaft

- Veteranenstatus

- Abhängigkeit vom Sozialsystem

- Behinderung

- Familienstand

- anderem

Im Manual wird anhand von ProblemBeispielen eine Handreichung für die Klassifizierung gegeben. Die jeweilige Umkehrung ließe sich als implizite Norm verstehen. So werden beispielsweise als mögliche Probleme bei »ökonomisches System und System der Grundversorgung, Arbeit» genannt:

- Arbeitslosigkeit aufgrund von mangelndem Arbeitsplatzangebot in einer Gemeinde

- Unterbeschäftigung (z. B. in einer Gemeinde gibt es nur Teilzeit-Jobs oder Niedriglohn-Jobs)

- keine adäquate Beschäftigung (keine sozial und legal akzeptable Beschäftigungsmöglichkeit, z. B. der Handel mit Drogen ist die einzig mögliche Einkommensquelle in einer Gemeinde)

In einer Umkehrung könnte die Norm so formuliert werden: In einer Gemeinde sollten ausreichend sozial akzeptable legale Arbeitsplätze (Vollzeit wie Teilzeit) mit angemessenem Lohn für alle Bevölkerungsgruppen ohne Diskriminierung zur Verfügung stehen.

Eine Skalierung erfolgt mit den Indizes für Intensität und Dauer des identifizierten Problems. Die Skala der Intensität reicht von 1 (kein Problem) bis 6 (katastrophal), jene für die Dauer von 1 (mehr als fünf Jahre) bis 6 (weniger 
als zwei Wochen).

Die Möglichkeit, Kommunikationspartnerinnen und Kommunikationspartner zu finden, ist heutzutage durch das Internet entschieden ausgeweitet worden. Wer vor Ort keine Gleichgesinnten findet, kann - den Zugang zum World Wide Web vorausgesetzt - Austauschpartner auch fern des eigenen Wohngebiets finden. Auch wenn die Qualität dieser Beziehungen mit jener einer subkulturellen Gruppe vor Ort nicht mithalten kann, bietet sie doch eine Chance, Gemeinschaft und Bestätigung zu erfahren, mit den eigenen Vorstellungen und Problemen nicht allein zu sein. Die Möglichkeit zur Nutzung des Internets müsste heute jedenfalls in ein solches Umweltassessment aufgenommen werden.

\section{Sozialraumbezug und Person- In-Environment System}

Es ist offensichtlich, dass das Person-InEnvironment System durch die Thematisierung der Umweltqualität des vorfindlichen Gemeinwesens den Blick auf den Sozialraum begünstigt. Auch hier stehen allerdings noch von der Sozialarbeit - vor allem der personenbezogenen, für die das Person-In-Environment System entwickelt wurde - wenig beeinflussbare Faktoren im Vordergrund. So gelangen zwar ungünstige Umweltbedingungen in den Fokus, eine Schiene zu sozialraumbezogenen Interventionen wird aber noch nicht erkennbar gelegt.

Das größte Potenzial hat Sozialarbeit bei der Förderung der Responsivität von zivilgesellschaftlichen Akteuren auf benachteiligte Personen und Personengruppen sowie beim Aufbau und der Verbesserung von Person-PersonNetzwerken (EGO-centered networks). Gerade diese beiden Systeme werden im Person-In-Environment System nur unzureichend abgebildet.

Fallunspezifische Arbeit im Gemeinwesen könnte allerdings unter den oben genannten Voraussetzungen von der Anwendung des Person-In-Environment Systems (in gegebenenfalls adaptierter Form) und einer statistischen Auswertung profitieren. Die Schwachstellen von Gemeinwesen könnten so dokumentiert und die Dokumentation zur Grundlage von gestaltender und Lobby-Arbeit gemacht werden.

\section{Literatur}

Adler, Helmut (1998): Eine gemeinsame Sprache finden. Klassifikation in der Sozialen Arbeit. Ein Versuch: das Person-In-Environment System (PIE). In: Blätter der Wohlfahrtspflege 7+8. S. $161-164$.

Adler, Helmut (2003): Person in Environment (PIE). Vorteile einer eigenen standardisierten Diagnostik in der Sozialen Arbeit. In: Heiner, Maja (Hg.): Diagnostik und Diagnosen in der Sozialen Arbeit. Frankfurt am Main.

Ehlerding, Marc (2002): Sind Klassifikationen sinnvoll? Zur Anwendbarkeit des Person-In-Environment Systems in der klinischen Sozialarbeit. Diplomarbeit. Norderstedt.

Karls, J. M./Wandrei, K. E. (1992): PIE - A NeW Language for Social Work. In: Social Work Nr. 37. Washington DC. S. 80-86

Karls, J. M./Wandrei, K. E. (Eds.) (1994):

PIE-Manual. Person-In-Environment System. Washington. Deutsche Übersetzung (2007): Internet http://www.pantucek.com/sozialediagnostik/verfahren/70-person-in-environment-classification-system-pie.html.

Karls. James M./O’Keefe, Maura E. (2008):

Person-In-Environment System Manual 2nd Edition. Washington.

Löcherbach, Peter (2010): Assessment im Case Management und sozialpädagogische Diagnostik. In: Schrapper, Christian (Hg.): Sozialpädagogische Diagnostik und Fallverstehen in der Jugendhilfe. Anforderungen - Konzepte - Perspektiven. 2. Auflage. Weinheim. S. 69-84. Mandiberg, James M./Miyaoka, Kyoko (1994): Using the PIE System to Teach Social Work Skills in Japan. In: Karls, James M./Wandrei, Karin E. (ed.): Person-in-Environment system: the PIE classification system for social functioning problems. Washington DC (USA): NASW press. S. $113-128$.

Pantucek, Peter (2009): Soziale Diagnostik. Verfahren für die Praxis Sozialer Arbeit. 2., verbesserte Auflage. Wien, Köln, Weimar. Pearson, Richard E. (1997): Beratung und soziale Netzwerke. Eine Lern- und Praxisanleitung zur Förderung sozialer Unterstützung. Weinheim und Basel.

Schrödter, Mark (2005): Wer macht bessere Diagnosen: Der Mensch oder die Maschine. Zu den interventionspraktischen Möglichkeiten und Gefahren diagnostischer Klassifikationssysteme. In: Manuskript. Bielefeld.

Schrödter, Mark (2009): Formalisierte Diagnostik, ja, aber richtig!. In: Pantucek, Peter/Röh, Dieter (Hg.): Perspektiven Sozialer Diagnostik. Wien, Münster. S. 57-78.

Warren, Roland (1963): The Community in America, 3rd ed. Chicago (USA).

\section{Gut beraten.}

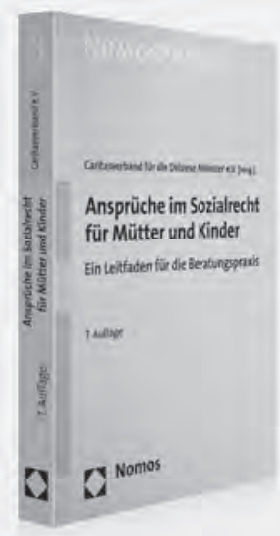

\section{Ansprüche im Sozialrecht für Mütter und Kinder}

Ein Leitfaden für die Beratungspraxis

Herausgegeben vom Caritasverband für die Diözese Münster e.V.

Verfasst von: RAin Birgit Scheibe 7. Auflage 2012, ca. $300 \mathrm{~S}$., brosch., ca. 24,- $€$, ISBN 978-3-8329-5969-2

Erscheint ca. April 2012

Auf aktuellem Stand und natürlich unter Berücksichtigung der grundlegenden Änderungen durch die Hartz-IV-Reform sowie der Änderungen beim Elterngeld und in der Krankenversicherung bietet die Neuauflage des Leitfadens allen Sozialrechtsberatern/innen systematisch wie praxisorientiert Lösungen zu Fragen der juristischen Beratungspraxis. 